Received: 2016.10 .01
Accepted: 2016.12 .01

Published: 2017.08 .10

\section{Percutaneous Transhepatic Biliary Stenting with Uncovered Self-Expandable Metallic Stents in Patients with Malignant Biliary Obstruction - Efficacy and Survival Analysis}

Authors' Contribution:
A Study Design
B Data Collection
C Statistical Analysis
D Data Interpretation
E Manuscript Preparation
F Literature Search
G Funds Collection

\author{
Andrius Pranculis ${ }^{1 \mathrm{ADEF}}$, Mantas Kievišas ${ }^{2 \mathrm{ABCDEF}}$, Lina Kievišienè ${ }^{2 \mathrm{ABCDEF}}$, \\ Artūras Vaičius $^{3 \mathrm{ABCDE}}$, Tomas Vanagas ${ }^{3 \mathrm{ADE}}$, Rytis Stasys Kaupas ${ }^{1 \mathrm{ADD}}$, \\ Žilvinas Dambrauskas, 3 ,4ACDEF \\ ${ }^{1}$ Department of Radiology, Lithuanian University of Health Sciences, Kaunas, Lithuania \\ ${ }^{2}$ Faculty of Medicine, Medical Academy, Lithuanian University of Health Sciences, Kaunas, Lithuania \\ ${ }^{3}$ Department of Surgery, Lithuanian University of Health Sciences, Kaunas, Lithuania \\ ${ }^{4}$ Institute for Digestive System Research, Lithuanian University of Health Sciences, Kaunas, Lithuania \\ Author's address: Mantas Kievišas, Lithuanian University of Health Sciences, Faculty of Medicine, Medical Academy, \\ 2 Eiveniu st, Kaunas, LT-50009, Lithuania, e-mail: mantas.kiev@gmail.com
}

Background: The aim of this study was to assess short- and long-term outcomes of malignant biliary obstruction (MBO) treatment by percutaneous transhepatic biliary stenting (PTBS) with uncovered selfexpandable metallic stents (SEMS), and to identify predictors of survival.

Material/Methods: $\quad$ A nine-year, single-centre study from a prospectively collected database included 222 patients with inoperable MBO treated by PTBS with uncovered nitinol SEMS.

Results: $\quad$ Technical and clinical success rates were $95.9 \%$ and $82.4 \%$, respectively. The total rate of postprocedural complications was $14.4 \%$. The mean durations of the primary and secondary stent patency were $114.7 \pm 15.1$ and $146.4 \pm 21.2$ days, respectively. The 30 -day mortality rate was $15.3 \%$ with no procedure-related deaths. The mean estimated length of survival was $143.3 \pm 20.6$ days. Independent predictors increasing the risk of death included higher than $115 \mu \mathrm{mol} / \mathrm{L}$ serum bilirubin 2-5 days after biliary stenting (HR 3.274, $P=0.019$ ), distal (non-hilar) obstruction of the bile ducts (HR 3.711, $P=0.008$ ), Bismuth-Corlette type IV stricture (HR 2.082, $P=0.008$ ), obstruction due to gallbladder cancer (HR 31.029, $P=0.012$ ) and only partial drainage of liver parenchyma (HR 4.158, $P=0.040$ ).

Conclusions:

PTBS with uncovered SEMS is an effective and safe method for palliative treatment of MBO. Serum bilirubin higher than $115 \mu \mathrm{mol} / \mathrm{L} 2-5$ days after the procedure has a significant negative impact on patients' survival. Lower survival is also determined by distal bile duct obstruction, BismuthCorlette type IV stricture, biliary obstruction caused by gallbladder cancer and when only partial liver drainage is applied.

MeSH Keywords: $\quad$ Cholestasis • Gastrointestinal Neoplasms • Radiology, Interventional • Stents • Survival Analysis

PDF file: $\quad$ http://www.polradiol.com/abstract/index/idArt/901785

\section{Background}

Malignant biliary obstruction (MBO) can be caused by primary tumours (most frequently cholangiocarcinomas, gallbladder cancer and pancreatic carcinomas), metastases or compression of metastatic lymph nodes [1].
At the time of diagnosis, for the majority of patients, curative surgical treatment is not possible and the median survival time is short. Hence, palliative treatment, which is aimed at providing drainage of the obstructed ductal system, is most frequently applied [2-4]. 
Inoperable MBO is palliatively treated by performing a surgical bilioenteric bypass or by using minimally invasive methods such as percutaneous transhepatic biliary drainage (PTBD), endoscopic biliary stenting (EBS) or percutaneous transhepatic biliary stenting (PTBS). EBS constitutes the treatment of choice, while PTBS is most frequently applied in cases when EBS fails or is not technically feasible [5]. In addition, PTBS is the preferred method in cases of proximal (hilar) strictures (obstruction appearing at or around the bile duct confluence) [6]. Although PTBS can be performed with either plastic stents (PS) or self-expandable metallic stents (SEMS), the benefit of the latter is manifested by higher rates of successful drainage and longer survival [7].

It is clear from the field studies conducted so far that PTBS is an effective and safe procedure of palliation for patients with MBO. However, there are only few articles analysing predictors of survival. In addition, previous studies that analysed patient survival present outcomes that are controversial, and there is as yet no general agreement regarding either the technique of the procedure or the selection of patients.

The aim of our study was to assess short- and long-term outcomes of malignant biliary stricture treatment by percutaneous transhepatic bile duct stenting with uncovered self-expandable metallic stents, and to identify predictors of patient survival.

\section{Material and Methods}

\section{Patient population}

The study included 222 patients treated by PTBS with uncovered nitinol SEMS. The indication for the percutaneous stenting was an inoperable malignant symptomatic biliary obstruction. Clinical and epidemiological characteristics of the enrolled patients are presented in Table 1.

\section{Data collection and analysis}

The study protocol was approved by the Institutional Ethics Committee (No. BEC-MF-240). From January 2007 to January 2016, data concerning PTBS procedures that were carried out in our institution were prospectively accumulated. Data on poststenting complications, stent patency and patient survival were collected retrospectively from patients' medical records and the National Cancer Registry.

The main objective of our study was to identify the predictors of survival, including, age and sex, the disease leading to the biliary stricture, the level of bile duct obstruction, the type of liver parenchyma drainage (whole versus partial), the type of procedure (two-stage vs. one-stage), the level of serum bilirubin before and within 2-5 days after the intervention and the presence of postprocedural complications. Moreover, technical and clinical success of PTBS as well as stent patency duration, and the data on the repeated stenting and indications for it were analysed.

\section{Technique}

Twelve hours before the procedure and 48-72 hours after it, prophylactic intravenous antibiotics (cefuroxime $1.5 \mathrm{~g}$, metronidazole $500 \mathrm{mg}$ ) were administered as part of a routine protocol. Before the procedure, the patient's blood clotting was examined, and in cases of clinically significant coagulopathy (an international normalized ratio [INR] greater than 1.5), stenting was not performed. Stents were inserted by a single-stage procedure in the presence of a medical team comprising of an interventional radiologist and abdominal surgeon. The procedures were performed with ultrasound and fluoroscopic guidance, with the patient under local anaesthesia and moderate (conscious) sedation. In some cases (in the presence of cholangitis, sepsis, interventional radiology suite not readily available for procedure, etc.), placement of the percutaneous cholangiostomy (external biliary drainage catheter) under ultrasound guidance by the surgeon and stenting under fluoroscopic

Table 1. Characteristics of patients $(\mathrm{n}=222)$ in the study group.

\begin{tabular}{|c|c|c|c|}
\hline Age & & \multicolumn{2}{|c|}{69.2 (13.1) years (range 26-99) } \\
\hline Males & & \multicolumn{2}{|c|}{$\mathrm{n}=93(41.9 \%)$} \\
\hline Patients with PTCS prior to stenting & & \multicolumn{2}{|c|}{$\mathrm{n}=172(77.5 \%)$} \\
\hline \multicolumn{2}{|c|}{ Type of tumour } & \multicolumn{2}{|c|}{ Level of obstruction } \\
\hline Pancreatic cancer & $\mathrm{n}=64(28.8 \%)$ & Proximal & $\mathrm{n}=145(65.3 \%)$ \\
\hline Cholangiocarcinoma & $\mathrm{n}=56(25.2 \%)$ & Type I & $\mathrm{n}=69(31.1 \%)$ \\
\hline Metastatic tumours in liver or LN & $\mathrm{n}=51(23 \%)$ & Type II & $\mathrm{n}=22$ (9.9\%) \\
\hline Gastric cancer & $\mathrm{n}=22(9.9 \%)$ & Type Illa & $n=16(7.2 \%)$ \\
\hline Gallbladder cancer & $\mathrm{n}=15(6.8 \%)$ & Type IIIb & $\mathrm{n}=21(9.5 \%)$ \\
\hline Hepatocellular carcinoma & $\mathrm{n}=7(3.2 \%)$ & Type IV & $\mathrm{n}=17(7.7 \%)$ \\
\hline Papilla Vater cancer & $\mathrm{n}=7(3.2 \%)$ & Distal & $\mathrm{n}=77(34.7 \%)$ \\
\hline
\end{tabular}




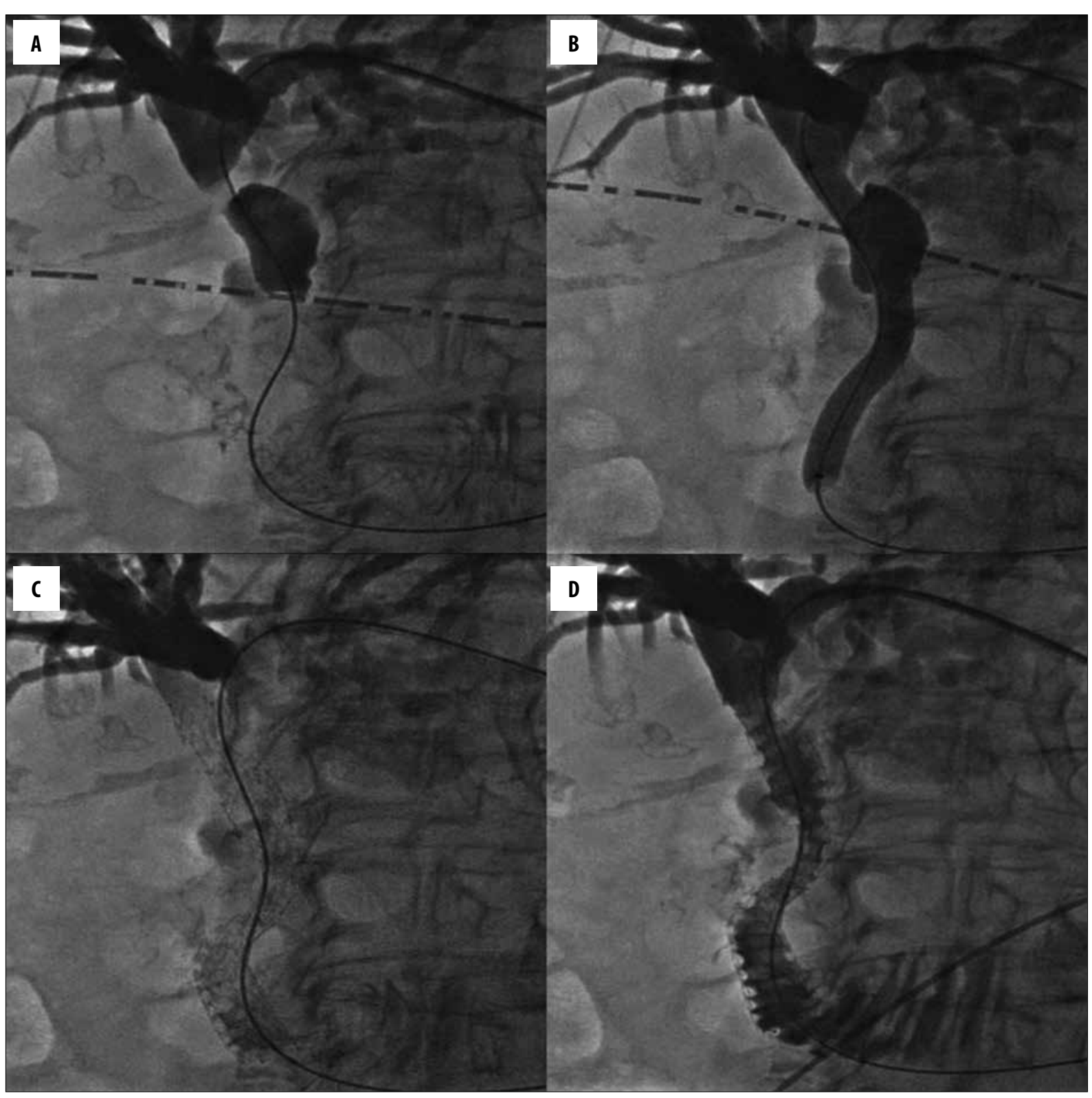

Figure 1. Percutaneous transhepatic cholangiogram demonstrating a Bismuth-Corlette type I biliary obstruction (A), dilatation of the stricture with a balloon catheter that was pulled over the guidewire $(\mathbf{B})$, stent insertion over a guidewire $(\mathbf{C})$, and final fluoroscopic image demonstrating the flow of contrast medium into the duodenum (D).

guidance by the interventional radiologist were performed as a two-stage procedure.

Depending on the tumour size and its localization, the right intercostal and/or left epigastric access was chosen. The initial percutaneous transhepatic bile duct puncture was performed with a 21-gauge needle. Then, with the help of different catheters and dilators, the 0.035 -inch guidewire was advanced through the bile duct stricture into the duodenum. Before stent insertion, the stricture (occlusion) was dilated with a balloon catheter that was pulled over the guidewire. Later, the catheter was removed, and the stent was inserted with the help of the same wire (Figure 1A-1D). In all cases, uncovered self-expandable metallic (nitinol) stents, including Epic ${ }^{\circledR}$ (Boston Scientific Corp., Natick, Massachusetts, USA), Protege ${ }^{\circledR}$ EverFlex $^{\circledR}$
(EV3, Plymouth, Minnesota, USA), Absolute Pro ${ }^{\circledR}$ (Abbott Vascular, Santa Clara, California, USA) and Misago ${ }^{\circledR}$ (Terumo Europe NV, Leuven, Belgium), were used. The strictures of the common bile and common hepatic ducts were most frequently stented with $10-\mathrm{mm}$ diameter stents, and the strictures of the lobar ducts (right and left hepatic ducts) were stented with 7-8-mm diameter stents. The length of the stent was chosen according to the length of the lesion. Furthermore, in order to avoid overgrowth of the stent by the tumour bulk, we made use of prevailing recommendations to leave a "safe location zone" of at least $2 \mathrm{~cm}$ equally over the upper and below the lower stricture pole when stenting [8]. On average, the procedure (cholangiography with PTBS) lasted 45.5 (27.7) minutes. 


\section{Technical and clinical success}

The intervention was considered technically successful if the stent expanded at the intended location and provided successful bile drainage through the stented duct(s). The procedure was considered to be clinically successful if during the first 2-5 days after stenting, the total serum bilirubin level had decreased by at least $10-15 \%$ in comparison to the initial level [9].

\section{Complications}

According to the guidelines of the Society of Interventional Radiology [10], the postprocedural complications were grouped into minor (do not cause long-term effects, treatment is not necessary or, if necessary, is either minimal or involves only observation) and major (cause long-term adverse effects or death and require hospitalisation and treatment). Minor complications include bleeding with no need for haemotransfusion, biliovenous fistula and subcapsular bilioma. Major complications comprise acute haemobilia, pancreatitis, pneumothorax, sepsis, liver abscess, cholecystitis, biliary peritonitis, bleeding requiring haemotransfusion, common bile duct rupture and cholangitis. Complications were evaluated during the first 30 days after the procedure.

\section{Stent patency and survival}

For the analysis of survival and stent patency duration, the Kaplan-Meier method was used, and the outcomes are presented with a standard error. The patient survival assessment period lasted from the date of the procedure until either death or the end of the study. Primary stent patency was defined as the period of time (expressed in days) when the inserted stents remained patent without any additional intervention; secondary stent patency was defined as the period of time (expressed in days) when stents remained patent following one additional re-intervention (percutaneous or endoscopic). Stents were considered patent in cases in which no increase in serum bilirubin level or no dilatation of intrahepatic ducts was registered. If obstruction or dislocation of the stent was not evident during the rest of the patient's lifetime, stent patency duration was considered to be equal to the survival length.

\section{Level of biliary obstruction}

The lesions were defined as proximal (pertaining to the hilar area, "hilar") if the tumours were above the cystic duct entrance location, and distal ("non-hilar") if they were below that point. The type of the proximal duct stricture was determined according to the Bismuth-Corlette classification.

\section{Unilobar vs. bilobar stenting and whole-liver vs. partial-liver drainage}

When analysing the method of liver parenchyma drainage, the patients were divided into two groups, as in the study conducted by Brountzos et al. [4]: 1) the partial-liver drainage group which included patients with Bismuth-Corlette type II to IV obstructions treated by unilobar (unilateral) stenting; and 2) the whole-liver drainage group, comprising of patients with distal strictures, Bismuth-Corlette type I lesions and Bismuth-Corlette type II to IV obstructions treated by bilobar (bilateral) stenting.

\section{One-stage vs. two-stage procedure}

Patients were also divided into two-stage and one-stage groups. The first group comprised of patients who were treated by percutaneous transhepatic cholangiostomy (PTCS) prior to biliary stenting, while the second group included patients who were treated by direct stenting.

\section{Statistical analysis}

The statistical analysis was performed using the SPSS 22.0 statistical package (IBM, Armonk, New York, USA). The normality of data distribution was checked using the Kolmogorov-Smirnov and Shapiro-Wilk tests. Normally distributed data are presented as means (standard deviation), and were compared with the paired and unpaired Student's t-tests. Non-parametric data are presented as medians (interquartile range), and were compared using the MannWhitney U-test for independent samples and the Wilcoxon signed-rank test for paired data. Categorical variables were compared using the chi-square test $\left(\chi^{2}\right)$ and Fisher's exact test. Qualitative data are presented as absolute values and as percentages.

A receiver operating characteristic (ROC) curve was used for calculations of poststenting serum bilirubin cut-off value that best dichotomizes the positive and the negative patient outcomes (survival time $>60$ days, absence of major complications, clinical success of the procedure).

The univariate survival analysis was performed for the analysis of the impact of various factors upon patient survival. The log-rank (Mantel-Cox) test was used to determine statistically significant differences between the groups. In addition, multivariate analysis was performed using the Cox proportional hazards model to identify the independent predictors of survival.

The results were considered statistically significant at $P<0.05$.

\section{Results}

In total, $95.9 \%(n=213)$ of stenting cases were technically successful, while a positive therapeutic effect (clinical success) was attained in $82.4 \%(n=183)$ of cases. From 2 to 5 days after PTBS, the total serum bilirubin level decreased in $90.1 \%(n=200)$ of patients. The median total serum bilirubin level before stenting was $240 \mu \mathrm{mol} / \mathrm{L}$ (range 144.9-347), while it decreased to $123 \mu \mathrm{mol} / \mathrm{L}$ (range 60.3-190) 2-5 days after PTBS. Both total and direct serum bilirubin levels decreased significantly $(P<0.001)$, i.e., total bilirubin by $48.4 \%$ (range 18.4-66.2\%) and direct bilirubin by $53.9 \%$ (range $27.7-71.3 \%$ ).

The total rate of early poststenting complications was $14 \%$. The most frequent minor complications included bleeding with no need for haemotransfusion, while the most 
Table 2. Early ( $<30$ days) procedure-related complications.

\begin{tabular}{|c|c|}
\hline Major complications ( $n=22$ ) & $9.9 \%$ \\
\hline Sepsis $(n=8)$ & $3.6 \%$ \\
\hline Cholangitis ( $\mathrm{n}=7$ ) & $3.2 \%$ \\
\hline $\begin{array}{l}\text { Major bleeding (requiring haemotransfusion) } \\
(n=2)\end{array}$ & $0.9 \%$ \\
\hline Acute haemobilia ( $n=2$ ) & $0.9 \%$ \\
\hline Acute pancreatitis ( $n=2$ ) & $0.9 \%$ \\
\hline Liver abscess $(\mathrm{n}=1)$ & $0.5 \%$ \\
\hline Minor complications $(n=10)$ & $4.5 \%$ \\
\hline $\begin{array}{l}\text { Minor bleeding (without haemotransfusion) } \\
(\mathrm{n}=9)\end{array}$ & $4.1 \%$ \\
\hline Biliovenous fistula ( $n=1$ ) & $0.5 \%$ \\
\hline
\end{tabular}

common major complications were sepsis and cholangitis. The rates of complications which occurred after PTBS are presented in Table 2.

The mean duration of the estimated primary stent patency was $114.7 \pm 15.1$ days (range 0-780 days). Cumulative primary patency rates after one, three and six months were $74.2 \%, 41.9 \%$ and $24.9 \%$, respectively. The mean period of secondary stent patency was $146.4 \pm 21.2$ days. Cumulative secondary patency rates after one, three and six months were $81.4 \%, 47.3 \%$ and $30.4 \%$, respectively.

Re-stenting was performed in 25 (11.3\%) patients. In one patient, an additional stenting was performed twice. Stent occlusion (Figure 2A, 2B) was the most frequent cause for re-stenting and occurred in 16 patients $(7.2 \%)$. The period leading to stent occlusion ranged between 4 and 264 days (mean 81 days). Three (1.4\%) patients needed re-stenting because of stent dislocation, three (1.4\%) patients had to undergo an additional procedure because of a stricture in a different location. Four (1.8\%) patients needed re-stenting due to unsuccessful first PTBS procedure.

Thirty-four patients (15.3\%) died within 30 days (range 0-30 days, mean 12.1 days) of stent placement. One patient (0.5\%) died during the first 24 hours after stenting because of preprocedural cholangitis that started to progress and later developed into septic shock. No deaths occurred due to the stent insertion procedure. The causes of death within the first 30 days were as follows: poor condition due to advanced disease in 21 patients $(9.5 \%)$, sepsis and cholangitis in $9(4.1 \%)$ patients, myocardial infarction and pulmonary embolism in $2(0.9 \%)$ patients each.

The mean estimated length of survival was $143.3 \pm 20.6$ days (range 0-780 days), while the median survival duration was $74 \pm 10.2$ days. The overall survival rate of patients after 1, 3, 6 and 12 months was $81.7 \%, 47.3 \%$, $29.6 \%$ and $10.4 \%$, respectively. The univariate survival analysis revealed that patients who suffered from gallbladder and gastric cancer had the shortest mean estimated length of survival $(61.6 \pm 12.3$ days and $78.9 \pm 12.7$ days, respectively), while the longest survival was seen in patients diagnosed with cholangiocarcinoma (191.7 \pm 46.4 days). We calculated that the $115-\mu \mathrm{mol} / \mathrm{L}$ cut-off value of total serum bilirubin 2-5 days after PTBS best dichotomized the positive and the negative patient outcomes. Our analysis identified that survival of patients with postprocedural serum bilirubin levels higher than $115 \mu \mathrm{mol} / \mathrm{L}$ was $116.2 \pm 29.9$ days, compared to $183.5 \pm 33.5$ days when bilirubin levels were $115 \mu \mathrm{mol} / \mathrm{L}$ or lower $(P=0.005$; Figure 3A). The survival analysis also revealed that the mean survival time of patients was statistically significantly better when no complications occurred after stenting (221.9 \pm 75.8 days vs. $131.3 \pm 18$ days, $P=0.010$; Figure $3 \mathrm{~B}$ ). Univariate survival analysis of patients grouped according to other characteristics is discussed in separate paragraphs below. Multivariate analysis was performed with all factors that were used in the univariate analysis. Independent predictors that increased the risk of death included distal obstruction of bile ducts, Bismuth-Corlette type IV

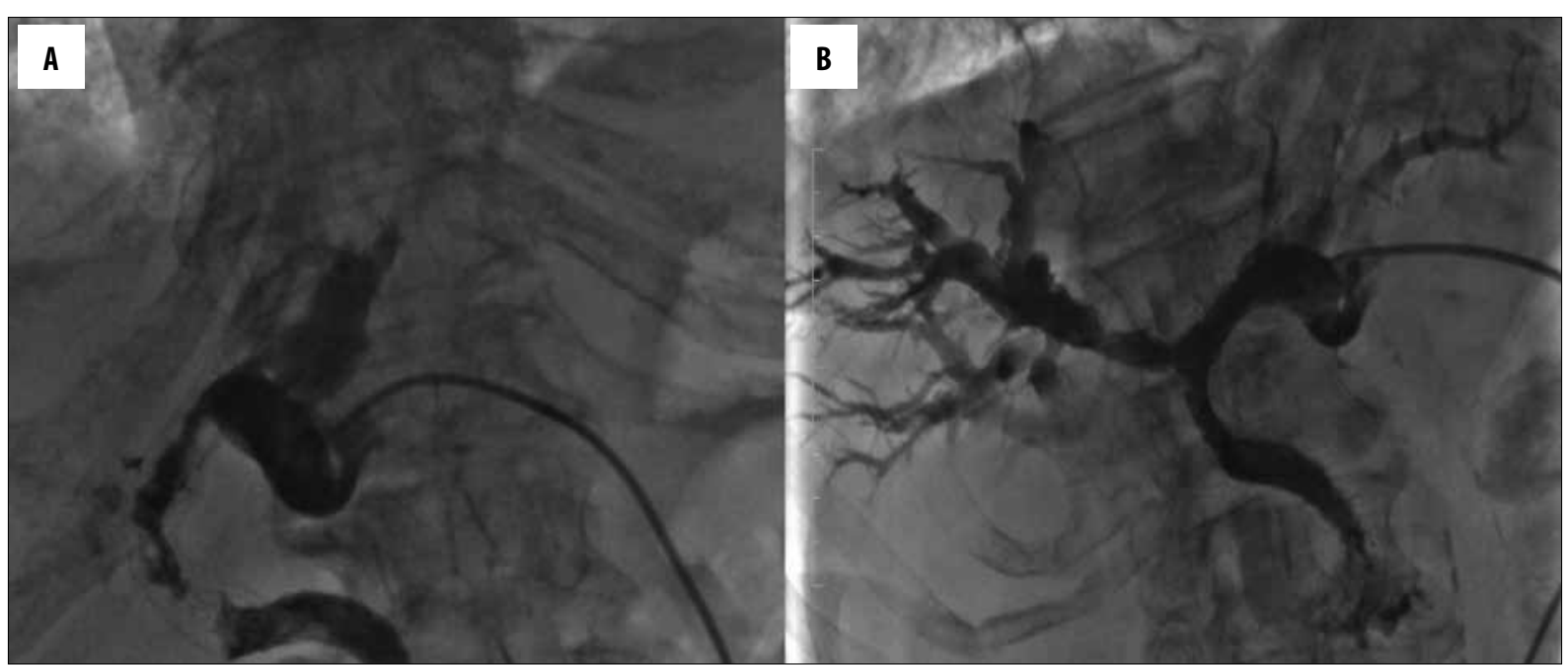

Figure 2. A case of an 86-year-old woman diagnosed with a cholangiocarcinoma. Percutaneous transhepatic cholangiogram demonstrating reocclusion of the stent in the left and common hepatic duct (A); final fluoroscopic image demonstrating the flow of contrast medium into the duodenum after T-configured bilateral stent implantation (B). 

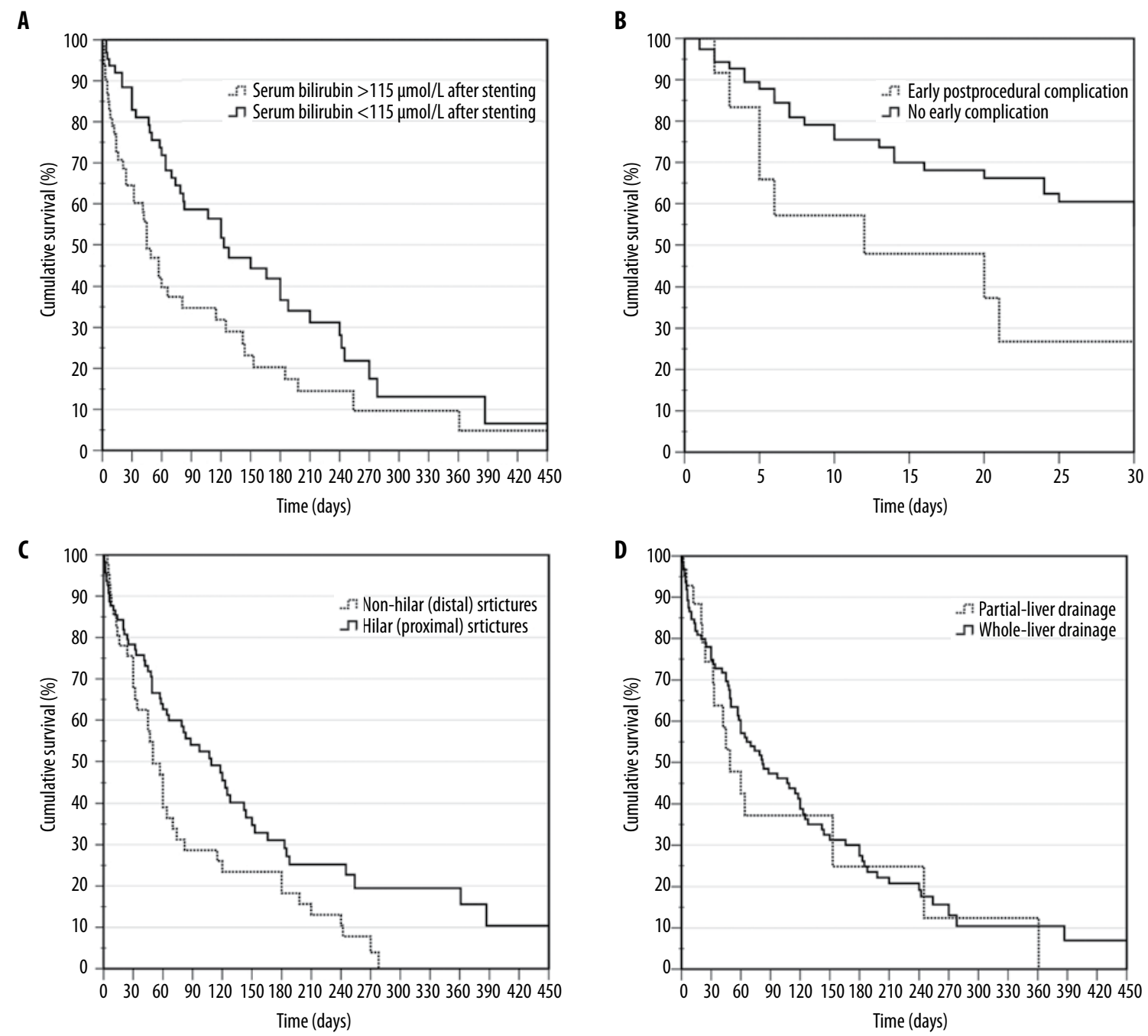

Figure 3. Overall survival with regard to: serum bilirubin levels after stenting $(\mathbf{A})$, occurrence of early $(<30$ days) procedure-related complications (B), level of biliary obstruction (C) and method of liver parenchyma drainage (D). Differences between curves were statistically significant in all cases $(P<0.05)$.

stricture, biliary obstruction due to gallbladder cancer, only partial drainage of liver parenchyma, and more than $115 \mu \mathrm{mol} / \mathrm{L}$ serum bilirubin 2-5 days after biliary stenting (Table 3).

When analysing the level of biliary obstruction, we identified that the mean survival time was considerably better in patients with proximal strictures compared to patients with distal strictures (186.4 \pm 33.4 days vs. 86.7 \pm 13.9 days, $P=0.005$; Figure 3C). Moreover, the mean primary stent patency time was also significantly longer when strictures were in proximal bile ducts $(142.8 \pm 24.6$ vs. $81.2 \pm 13.1$ days, respectively; $P=0.020$ ). Changes of serum bilirubin after stenting, primary stent patency and patient survival relating to the location of the bile duct obstruction are presented in Table 4.

When compared to unilobar stenting, a significantly longer mean patency time and survival duration were registered for patients with Bismuth-Corlette type III strictures treated by bilobar stenting (Table 4). Furthermore, a significantly greater bilirubin level decrease was identified in bilaterally stented patients compared to unilaterally stented patients with Bismuth-Corlette type IV obstruction (Table 4). In addition, there was no statistically significant difference between the rate of complications in unilobar and bilobar patient groups $(18.2 \%[\mathrm{n}=4]$ vs. $20.4 \%[\mathrm{n}=11]$, respectively; $P=0.828$ ). Considering the fact that obstructions that were treated by bilobar stenting were included into the whole-liver parenchyma drainage group, better outcomes were also obtained when comparing the wholeliver drainage group with the partial-liver drainage group (Figure 3D, Table 4).

The comparison of patients treated by PTCS before stenting (two-stage procedure) versus patients who were treated by direct stenting (one-stage procedure) with regard to the bilirubin percentage decrease did not establish a statistically significant difference $(P=0.353)$. The mean primary stent patency time for patients in the two-stage group was 
Table 3. Independent prognostic factors for survival after PTBS (multivariate survival analysis using Cox's regression model).

\begin{tabular}{|c|c|c|c|}
\hline & Hazard ratio & $\mathbf{9 5 \%}$ confidence interval & $P$ value \\
\hline Age & 1.023 & $0.999-1.049$ & 0.062 \\
\hline \multicolumn{4}{|l|}{ Sex } \\
\hline Male & Reference & - & - \\
\hline Female & 1.439 & $0.834-2.482$ & 0.191 \\
\hline \multicolumn{4}{|l|}{ Type of tumour } \\
\hline Cholangiocarcinoma & Reference & - & - \\
\hline Gallbladder cancer & 31.029 & $2.143-449.273$ & 0.012 \\
\hline Gastric cancer & 8.031 & $0.738-87.390$ & 0.087 \\
\hline Pancreatic cancer & 1.557 & $0.633-3.829$ & 0.335 \\
\hline Metastatic tumours in liver or LN & 1.560 & $0.559-4.352$ & 0.396 \\
\hline Hepatocellular carcinoma & 1.072 & $0.358-3.213$ & 0.901 \\
\hline Papilla Vater cancer & 1.014 & $0.325-3.164$ & 0.981 \\
\hline \multicolumn{4}{|l|}{ Level of biliary obstruction } \\
\hline Proximal ("hilar") & Reference & - & - \\
\hline Distal ("non-hilar") & 3.711 & $1.988-4.566$ & 0.008 \\
\hline \multicolumn{4}{|l|}{ Bismuth-Corlette classification } \\
\hline Type I & Reference & - & - \\
\hline Type II & 0.518 & $0.179-1.502$ & 0.226 \\
\hline Type III & 1.677 & $0.533-5.280$ & 0.377 \\
\hline Type IV & 2.082 & $1.208-3.589$ & 0.008 \\
\hline \multicolumn{4}{|c|}{ Application of PTCS prior stenting (two-stage procedure) } \\
\hline Yes & Reference & - & - \\
\hline No & 1.082 & $0.492-2.382$ & 0.844 \\
\hline \multicolumn{4}{|l|}{ Method of liver parenchyma drainage } \\
\hline Whole-liver & Reference & - & - \\
\hline Partial-liver & 4.158 & $1.84-20.588$ & 0.040 \\
\hline Serum bilirubin levels at presentation & 1.001 & $0.998-1.003$ & 0.500 \\
\hline \multicolumn{4}{|l|}{ Serum bilirubin 2-5 days after stenting } \\
\hline$<115 \mu \mathrm{mol} / \mathrm{L}$ & Reference & - & - \\
\hline$>115 \mu \mathrm{mol} / \mathrm{L}$ & 3.274 & $1.822-5.882$ & 0.019 \\
\hline \multicolumn{4}{|l|}{ Occurrence of early complications } \\
\hline Yes & Reference & - & - \\
\hline No & 1.925 & $0.793-4.675$ & 0.148 \\
\hline
\end{tabular}

LN - lymph nodes; PTCS - percutaneous transhepatic cholangiostomy.

$113.9 \pm 15.9$ days, while that of the one-stage group patients was $121.3 \pm 36.4$ days. The difference between the groups was not statistically significant $(P=0.949)$. Similar results were obtained when comparing the survival, $135.7 \pm 20.1$ days vs. $175.9 \pm 55.1$ days, respectively $(P=0.153)$.

\section{Discussion}

Our study analysed the impact of various factors upon the overall survival of patients and established that the independent predictors that increase the risk of death include distal obstruction of bile ducts, Bismuth-Corlette type IV stricture, biliary obstruction due to gallbladder cancer, only partial drainage of liver parenchyma and higher than $115 \mu \mathrm{mol} / \mathrm{L}$ serum bilirubin 2-5 days after biliary stenting. Indar et al. [11] examined the influence of baseline liver function tests on survival of patients and identified an inverse correlation only between serum bilirubin and overall survival. In contrast, our study revealed that patient survival was affected by high bilirubin levels not before but after the procedure. A study by Brountzos et al. [4] obtained findings that were similar to those in our study, i.e. the overall survival of patients was affected by high bilirubin level after PTBS (patients with serum bilirubin 
Table 4. Percentage changes in total bilirubin values after stenting, survival and primary stent patency duration in patients with different types of obstruction treated with unilobar or bilobar stenting and partial- or whole-liver drainage. Similar table design was used in a study by Brountzos et al. [4].

\begin{tabular}{|c|c|c|c|c|c|c|c|c|}
\hline $\begin{array}{l}\text { Obstruction } \\
\text { type }\end{array}$ & No. & \multicolumn{2}{|c|}{$\begin{array}{l}\text { Decrease in TBIL levels after } \\
\text { stenting, } \%\end{array}$} & \multirow{2}{*}{$\begin{array}{c}\begin{array}{c}P \\
\text { value }\end{array} \\
-\end{array}$} & \multirow{2}{*}{$\begin{array}{c}\text { Survival } \\
86.7 \pm 13.9\end{array}$} & \multirow{2}{*}{$\begin{array}{c}\begin{array}{c}P \\
\text { value }\end{array} \\
-\end{array}$} & \multirow{2}{*}{$\begin{array}{c}\text { Primary } \\
\text { patency }\end{array}$} & \multirow{2}{*}{$\begin{array}{c}\mathbf{P} \\
\text { value } \\
-\end{array}$} \\
\hline Distal & 77 & 52.4 & $(32.7-65.4)$ & & & & & \\
\hline Bismuth I & 69 & 52.9 & $(22-68.2)$ & - & $187.3 \pm 44$ & - & $155.3 \pm 35.1$ & - \\
\hline Bismuth II & 22 & 34.5 & (7.9-57) & \multirow{3}{*}{0.948} & $225.9 \pm 98.6$ & \multirow{3}{*}{0.340} & $161.9 \pm 77.1$ & \multirow{3}{*}{0.424} \\
\hline Unilobar stenting & 19 & 33.8 & $(27.6-52.2)$ & & $164.2 \pm 83$ & & $118.9 \pm 63.6$ & \\
\hline Bilobar stenting & 3 & 39.1 & $(19.2-58.3)$ & & 249.2 & & 176.9 & \\
\hline Bismuth III & 37 & 38.5 & $(8.8-60.6)$ & \multirow{3}{*}{0.357} & $134.4 \pm 23$ & & $106.9 \pm 21$ & \\
\hline Unilobar stenting & 25 & 37.2 & $(16.1-60.9)$ & & $98.1 \pm 28$ & \multirow{2}{*}{0.032} & $70 \pm 21.2$ & \multirow{2}{*}{0.007} \\
\hline Bilobar stenting & 12 & 43.4 & $(12.1-61.9)$ & & $200.7 \pm 98.1$ & & $178.8 \pm 22$ & \\
\hline Bismuth IV & 17 & 35.3 & $(2.2-72.7)$ & \multirow{3}{*}{$<0.001$} & $80.9 \pm 22.6$ & \multirow{3}{*}{0.257} & $65.8 \pm 20.8$ & \multirow{3}{*}{0.280} \\
\hline Unilobar stenting & 11 & 22.0 & $(7.4-67.3)$ & & $47 \pm 12$ & & $47 \pm 13.9$ & \\
\hline Bilobar stenting & 6 & 72.4 & $(65-82.6)$ & & $123 \pm 54.2$ & & $123 \pm 54.2$ & \\
\hline \multicolumn{9}{|c|}{ Method of liver parenchyma drainage } \\
\hline Partial-liver & 55 & 35.6 & $(0.5-60.9)$ & & $115.2 \pm 31.1$ & & $77.5 \pm 22.2$ & \\
\hline Whole-liver & 167 & 52.5 & $(26.2-70)$ & $<0.001$ & $153.2 \pm 24.6$ & 0.035 & $125.2 \pm 18$ & 0.044 \\
\hline
\end{tabular}

TBIL - total bilirubin. Percentage changes in bilirubin values after stenting are expressed as median (interquartile range). Survival and primary stent patency are expressed as estimated mean \pm standard error. The decrease in serum bilirubin levels was statistically significant for all types of obstruction according to the Bismuth-Corlette classification $(P<0.05)$.

levels of $68.4 \mu \mathrm{mol} / \mathrm{L}[4 \mathrm{mg} / \mathrm{dL}]$ or higher had a 3.64 times higher death risk) and by a Bismuth-Corlette type IV stricture. The same study revealed that the age of patients also influenced the overall survival; however, this finding is not consistent with our results.

It is widely accepted that distal (common bile duct) and Bismuth-Corlette type I (common hepatic duct) strictures should be stented with a single stent. However, currently there are no clear recommendations on whether single or multiple stents (unilobar or bilobar drainage) should be inserted for Bismuth type II or higher lesions. The current European Society of Gastrointestinal Endoscopy (ESGE) clinical guidelines for biliary stenting [12] as well as the Asia-Pacific consensus recommendations for endoscopic and interventional management of hilar cholangiocarcinoma [13] indicate that the aim of the stenting is to drain an adequate volume of liver (at least 50\%), irrespective of whether a bilobar or unilobar metal stenting is performed. Although the majority (72.4\%) of patients suffering from Bismuth types II-IV obstruction in our study were treated by unilobar stenting, more positive outcomes were established in the bilobar stenting group with regard to a more significant bilirubin level decrease after stenting (in patients with Bismuth IV obstruction) and higher stent patency as well as better patient survival time (in patients with Bismuth III strictures). In addition, there was no statistically significant difference between the rates of complications in each group. However, if compared to unilobar stenting, bilobar stenting was not always technically successful, while the cases of the former were all successful in our study (88.9\% vs. $100 \%$, $P=0.029$ ). Accordingly, better outcomes of bilobar stenting were reflected by better results in the whole-liver drainage group compared with the partial-liver drainage group.
The common practice followed in our hospital is to perform two-stage procedures, especially when signs of cholangitis and sepsis start to appear in patients who have MBO. In total, PTCS before stenting was performed in $77.5 \%$ of patients. However, the results obtained in our study revealed that neither patient survival time nor stent patency duration or the decrease in total serum bilirubin level benefited from PTCS performed before stenting, which is similar to the results obtained by Inal et al. [14].

Analysis of stent patency and survival in our study revealed unexpected statistically significant differences between the groups of patients with proximal and distal strictures. The mean duration of stent patency and the mean estimated patient survival time were significantly higher in the group of patients with proximal strictures when compared to the group of patients with distal obstruction. A prospective long-term study by Lee et al. [15] obtained outcomes that were similar to those in our study, i.e. the stent patency duration was significantly longer in the patients with hilar (proximal) strictures (617 vs. 324 days, $P<0.05)$. Patient survival was also slightly better in the group of patients with hilar (proximal) strictures; however, the difference was not statistically significant ( 257 vs. 227 days, $P>0.1$ ). In addition, several other recent studies identified a slightly higher rate of survival in the group of patients with proximal strictures; nevertheless, the differences between the groups were again statistically insignificant $[4,9]$. There are several aspects to explain with regard to the better outcomes obtained in the proximal patient group in our study. Firstly, the common practice followed in our hospital in cases of distal MBO is to perform endoscopic biliary stenting (EBS); meanwhile, PTBS is usually performed in patients for whom EBS had 
failed, most frequently due to advanced disease or tumour overgrowth. With regard to the fact that the majority of patients suffered from advanced distal $\mathrm{MBO}$, adequate bile drainage could be attained only with two (unsuccessful EBS followed by PTBS) or even three (unsuccessful EBS followed by PTCS and eventually PTBS) interventions. Consequently, the survival time of patients in the distal group might have been affected by potentially more advanced disease, longer period required for the reconstruction of adequate bile drainage and numerous interventions. Furthermore, in cases of bilaterally stented proximal obstructions, if one of the two stents becomes occluded, jaundice usually does not occur at once as the bile may drain through the other side. In contrast, in cases of distal strictures, if the only stent becomes occluded, jaundice occurs immediately [15]. These causes may also have an impact on patient survival and stent patency.

In most cases, the uncovered metallic stents recurrently occlude due to the formation of biliary sludge and tumour overgrowth or ingrowth into the stent. In our study, stent occlusion was confirmed in only $7 \%$ of patients. The mean duration of the primary state patency was 114.7 days. Our findings are consistent with the data in the literature, i.e. re-occlusion rate of $6-42 \%$ and mean stent patency time of 82-382 days [8,15-17]. These outcomes indicate that biliary stenting with uncovered SEMS is associated with reasonably low rates of stent migration and fair patency durations. Therefore, it is a suitable palliative option for patients with MBO because the majority of stents tend to remain patent at the time of the patient's death and thus re-stenting and additional procedures are rarely required.

The vast majority of studies show that percutaneous insertion of uncovered SEMS is technically successful in more than $95 \%$ of cases, and it is quite often indicated that the success rate is $100 \%[4,17-23]$. Successful bile drainage and a significant decrease in serum bilirubin levels have been reported as being between $71 \%$ and $98 \%[3,24]$. Findings of other studies demonstrate that procedure-related and early ( $<30$ days) mortality rates after stenting are $0-6 \%$ and $0-39 \%[2,5,20-22,25]$, respectively. Results from the reviewed studies, as well as those achieved in the present study, reveal that PTBS is a potentially safe and effective palliative procedure. This is very important as patients with inoperable MBO have a short remaining life expectancy and therefore the main treatment goals for these patients include relief of symptoms, improvement of their health-related quality of life, early discharge from hospital and, as a result, prevention of complications and repeated hospitalisations.

Our study has several limitations. Firstly, this study is retrospective in nature and was carried out in only one centre. Furthermore, PTBS indications that could have been analysed as a factor affecting patients' survival were not documented. In addition, the number of patients in the bilobar stenting group was quite small.

\section{Conclusions}

Our study confirmed that PTBS with uncovered SEMS is an effective and safe method of palliative treatment of patients with intrahepatic or extrahepatic bile duct obstruction caused by locally advanced primary or metastatic tumours. Moreover, we identified that the bilirubin level before stenting has no significant effect upon patient survival. However, a serum bilirubin level higher than 115 $\mu \mathrm{mol} / \mathrm{L} 2-5$ days after PTBS has a significant dismal impact on survival. Lower patient survival time is also determined by distal obstruction of the bile ducts, Bismuth-Corlette type IV strictures and biliary obstruction due to gallbladder cancer. We also determined that the whole-liver drainage method, in comparison to partial-liver drainage, as well as bilobar stenting compared to unilobar stenting, demonstrate better outcomes with regard to a more significant decrease in the bilirubin level after stenting and higher stent patency duration, together with longer patient survival time. In addition, our study established that neither patient survival time nor stent patency duration or a decrease in total serum bilirubin level benefit from percutaneous transhepatic cholangiostomy performed before stenting.

\section{References:}

1. Bae JI, Park AW, Choi SJ et al: Crisscross-configured dual stent placement for trisectoral drainage in patients with advanced biliary hilar malignancies. J Vasc Interv Radiol, 2008; 19: 1614-19

2. Thornton RH, Frank BS, Covey AM et al: Catheter-free survival after primary percutaneous stenting of malignant bile duct obstruction. Am J Roentgenol, 2011; 197: W514-18

3. Pinol V, Castells A, Bordas JM et al: Percutaneous self-expanding metal stents versus endoscopic polyethylene endoprostheses for treating malignant biliary obstruction: Randomized clinical trial. Radiology, 2002; 225: 27-34

4. Brountzos EN, Ptochis N, Panagiotou I et al: A survival analysis of patients with malignant biliary strictures treated by percutaneous metallic stenting. Cardiovasc Intervent Radiol, 2007; 30: 66-73

5. Ahn SJ, Bae JI, Han TS et al: Percutaneous biliary drainage using open cell stents for malignant biliary hilar obstruction. Korean J Radiol, 2012; 13: 795-802

6. Ray CE, Lorenz JM, Burke CT et al: ACR Appropriateness Criteria radiologic management of benign and malignant biliary obstruction. J Am Coll Radiol, 2013; 10: 567-74
7. Sangchan A, Kongkasame W, Pugkhem A et al: Efficacy of metal and plastic stents in unresectable complex hilar cholangiocarcinoma: A randomized controlled trial. Gastrointest Endosc, 2012; 76: 93-99

8. Lameris JS, Stoker J, Nijs HG et al: Malignant biliary obstruction: Percutaneous use of self-expandable stents. Radiology, 1991; 179: 703-7

9. Inal M, Akgul E, Aksungur E et al: Percutaneous self-expandable uncovered metallic stents in malignant biliary obstruction. Complications, follow-up and reintervention in 154 patients. Acta Radiol, 2003; 44: 139-46

10. Sacks D, McClenny TE, Cardella JF, Lewis CA: Society of Interventional Radiology clinical practice guidelines. J Vasc Interv Radiol, 2003; 14: S199-202

11. Indar AA, Lobo DN, Gilliam AD et al: Percutaneous biliary metal wall stenting in malignant obstructive jaundice. Eur J Gastroenterol Hepatol, 2003; 15: 915-19

12. Dumonceau JM, Tringali A, Blero D et al: Biliary stenting: indications, choice of stents and results: European Society of Gastrointestinal Endoscopy (ESGE) clinical guideline. Endoscopy, 2012; 44: 277-98 
13. Rerknimitr R, Angsuwatcharakon P, Ratanachu-ek T et al: Asia-Pacific consensus recommendations for endoscopic and interventional management of hilar cholangiocarcinoma. J Gastroenterol Hepatol, 2013; 28: 593-607

14. Inal M, Aksungur E, Akgul E et al: Percutaneous placement of metallic stents in malignant biliary obstruction: One-stage or twostage procedure? Pre-dilate or not? Cardiovasc Intervent Radiol, 2003; 26 : $40-45$

15. Lee $\mathrm{BH}$, Choe $\mathrm{DH}$, Lee JH et al: Metallic stents in malignant biliary obstruction: prospective long-term clinical results. Am J Roentgenol 1997; 168: 741-45

16. Brountzos EN, Petropoulos E, Kelekis NL et al: Malignant biliary obstruction: management with percutaneous metallic stent placement. Hepatogastroenterology, 1999; 46: 2764-71

17. Liu F, Zhang CQ, Wang GC et al: Percutaneous biliary stent placement in palliation of malignant bile duct obstruction. Gastroenterol Res, 2009; 2: 289-94

18. Schima W, Prokesch R, Osterreicher C et al: Biliary Wallstent endoprosthesis in malignant hilar obstruction: Long-term results with regard to the type of obstruction. Clin Radiol, 1997; 52: 213-19

19. Gwon D Il, Ko GY, Kim JH et al: A comparative analysis of PTFEcovered and uncovered stents for palliative treatment of malignant extrahepatic biliary obstruction. Am J Roentgenol, 2010; 195: W463-69
20. Krokidis M, Fanelli F, Orgera G et al: Percutaneous palliation of pancreatic head cancer: Randomized comparison of ePTFE/FEP covered versus uncovered nitinol biliary stents. Cardiovasc Intervent Radiol, 2011; 34: 352-61

21. Lawson AJ, Beningfield SJ, Krige JE et al: Percutaneous transhepatic self-expanding metal stents for palliation of malignant biliary obstruction. S Afr J Surg, 2012; 50: 54, 56, 58 passim

22. Karnabatidis D, Spiliopoulos S, Katsakiori P et al: Percutaneous trans-hepatic bilateral biliary stenting in bismuth IV malignant obstruction. World J Hepatol, 2013; 5: 114-19

23. Inal M, Akgul E, Aksungur E, Seydaoglu G: Percutaneous placement of biliary metallic stents in patients with malignant hilar obstruction: Unilobar versus bilobar drainage. J Vasc Interv Radiol 2003; 14: 1409-16

24. Kaskarelis IS, Papadaki MG, Papageorgiou GN et al: Long-term follow-up in patients with malignant biliary obstruction after percutaneous placement of uncovered wallstent endoprostheses. Acta Radiol, 1999; 40: 528-33

25. Han YH, Kim MY, Kim SY et al: Percutaneous insertion of Zilver stent in malignant biliary obstruction. Abdom Imaging, 2006; 31: 433-38 